\title{
Genomics of chromophobe renal cell carcinoma: implications from a rare tumor for pan-cancer studies
}

\author{
Kimryn W. Rathmell4,5,6, Fengju Chen ${ }^{1}$ and Chad J. Creighton ${ }^{1,2,3}$ \\ ${ }^{1}$ The Dan L. Duncan Cancer Center Division of Biostatistics, Houston, TX, USA \\ ${ }^{2}$ Department of Medicine, Baylor College of Medicine, Houston, TX, USA \\ ${ }^{3}$ Department of Bioinformatics and Computational Biology, The University of Texas M. D. Anderson Cancer Center, Houston, \\ TX, USA \\ ${ }^{4}$ Department of Urology, University of North Carolina, Chapel Hill, NC \\ ${ }^{5}$ Department of Genetics, University of North Carolina, Chapel Hill, NC \\ ${ }^{6}$ Department of Medicine, Division of Hematology and Oncology, University of North Carolina, Chapel Hill, NC \\ Correspondence to: Chad J. Creighton, email: creighto@bcm.edu \\ Keywords: ChRCC, chromophobe, kidney cancer, genomics, TERT, TCGA, mitochondria \\ Received: January 27, $2015 \quad$ Accepted: February 18, $2015 \quad$ Published: February 20, 2015
}

This is an open-access article distributed under the terms of the Creative Commons Attribution License, which permits unrestricted use, distribution, and reproduction in any medium, provided the original author and source are credited.

\section{ABSTRACT}

Chromophobe Renal Cell Carcinoma (ChRCC) is a rare subtype of the renal cell carcinomas, a heterogenous group of cancers arising from the nephron. Recently, The Cancer Genome Atlas (TCGA) profiled this understudied disease using multiple data platforms, including whole exome sequencing, whole genome sequencing (WGS), and mitochondrial DNA (mtDNA) sequencing. The insights gained from this study would have implications for other types of kidney cancer as well as for cancer biology in general. Global molecular patterns in ChRCC provided clues as to this cancer's cell of origin, which is distinct from that of the other renal cell carcinomas, illustrating an approach that might be applied towards elucidating the cell of origin of other cancer types. MtDNA sequencing revealed loss-of-function mutations in NADH dehydrogenase subunits, highlighting the role of deregulated metabolism in this and other cancers. Analysis of WGS data led to the discovery of recurrent genomic rearrangements involving TERT promoter region, which were associated with very high expression levels of TERT, pointing to a potential mechanism for TERT deregulation that might be found in other cancers. WGS data, generated by large scale efforts such as TCGA and the International Cancer Genomics Consortium (ICGC), could be more extensively mined across various cancer types, to uncover structural variants, mtDNA mutations, themes of tumor metabolic properties, as well as noncoding point mutations. TCGA's data on ChRCC should continue to serve as a resource for future pan-cancer as well as kidney cancer studies, and highlight the value of investigations into rare tumor types to globally inform principals of cancer biology.

\section{INTRODUCTION}

Chromophobe kidney cancer (ChRCC) is a rare subtype of renal cell carcinoma (RCC), a heterogenous group of cancers arising from the kidney nephron. As part of an effort by The Cancer Genome Atlas (TCGA), we comprehensively profiled 66 ChRCC cases at the molecular level [1]. This effort represented the first of a series of Rare Tumor Projects initiated by
TCGA, where each project comprehensively profiles a rare and understudied cancer subtype (other rare tumors currently under study including adrenocortical carcinoma, cholangiocarcinoma, paraganglioma and pheochromocytoma, sarcoma, thymoma, testicular germ cell tumors, uterine carcinoma, and uveal melanoma). Molecular data platforms involved in our study of ChRCC included whole exome sequencing, whole genome sequencing, mitochondrial DNA (mtDNA) sequencing, 
DNA methylation arrays, and mRNA and microRNA sequencing. This review revisits key findings made in this study, and further considers their implications for the study of other cancers.

Our study of ChRCC was unique in many respects. The multi-platform molecular data generated for this understudied disease, with the associated analysis work, was carried out at a level that would never have been possible from smaller scale studies. Even when compared to recent comprehensive, multi-platform studies of other cancers (from TCGA and others), ours represented the first such study featuring both a large number of both highcoverage whole genomes $(n=50$ cases, sequenced at a coverage of $60 \mathrm{X}$ read depth) and mtDNA sequences (by Long-range polymerase chain reaction or LR-PCR), where both data types were integrated with data from the other platforms. These data should help enable new thinking in areas relevant to many distinct, and perhaps more common cancers beyond kidney chromophobe. In particular, our findings pointed to a truly novel mechanism of TERT up-regulation in cancer (differing substantially from that of the activating point mutations reported elsewhere [2, 3]) and raise some provocative questions, regarding the precise role of mtDNA mutations, in cancers utilizing oxidative phosphorylation. Our study demonstrates that large scale molecular profiling of an understudied cancer can reveal novel cancer mechanisms, and can provide insights into the biology of even more common cancers.

\section{Chromophobe renal cell carcinoma (ChRCC) is a distinct disease}

ChRCC is one of the renal cell carcinomas, a heterogenous group of cancers arising from the kidney nephron. ChRCC is a rare tumor type accounting for approximately 5\% of RCC cases [4]. ChRCCs, characterized by a highly specific karyotype, exhibit an indolent pattern of local growth, with greater than $90 \%$ ten-year cancer-specific survival for localized disease [5, 6], but aggressive features and metastasis can occur. While chromophobe kidney cancer is associated with multiple cytogenetic abnormalities [7], detailed evaluation of the somatic genetics of this cancer had not been performed previously. ChRCC is seen at high frequency in BirtHogg-Dubé (BHD) syndrome, an autosomal dominant cancer predisposition syndrome due to mutations in $F L C N$, as nearly $40 \%$ of BHD-associated kidney tumors are characterized as ChRCC. However, FLCN mutations are rarely observed in sporadic ChRCC [8-10]. ChRCC has also recently been reported in Cowden syndrome, which is associated with PTEN mutations [11].

Only in the past two decades has it has been recognized that RCC represents a collection of highly distinct tumors, with distinct molecular and genetic features potentially reflecting the cell-of-origin as well as independent processes of tumorigenesis. Distinct molecular signatures may be seen in different cancer types, and it is not uncommon for cancers arising in different organs to have greater similarity than those belonging to a classic pathologic subtype within a single organ $[12,13]$. ChRCC was first described in 1985 as distinct from ccRCC because of unique morphologic features, including abundant cytoplasmic single-membrane vesicular structures that may arise from budding of the mitochondrial membrane $[14,15]$. An eosinophilic variant of ChRCC was subsequently identified with abundant mitochondria, resulting in the characteristic granular eosinophilic cytoplasmic staining, with few vesicular structures.

Our multi-platform analyses clearly confirmed that $\mathrm{ChRCC}$ is a disease entity distinct from the more common RCC, clear cell renal cell carcinoma (ccRCC). The molecular differences between ChRCC and ccRCC are easily highlighted by their characteristic features. For example, ChRCC lack the mutational events involving $V H L$ and chromatin remodeling genes on chromosome $3 \mathrm{p}$ that occur in the vast majority of ccRCC [16]. In contrast, ChRCC cases demonstrated a much larger percentage of TP53 mutations (32\% of the 66 cases) than are seen in ccRCC. ChRCC is characterized in part by a great degree of uniform chromosomal copy number alterations, with the majority of cases having loss of one copy of the entire chromosome, for most or all of chromosomes 1, 2, 6, 10, 13 , and 17 , while in ccRCC only the loss of chromosome $3 p$ is seen to a similar degree. Interestingly, a fraction of the both ccRCC and ChRCC ( 20\%) show alterations impacting the PTEN/MTOR pathway with the greatest number of mutations in ChRCC being present within the PTEN gene. Thus, the PTEN/MTOR pathway could represent a therapeutic target in at least some of these cancers that could be shared by both ChRCC and CCRCC tumors, though one important caveat here is that even the presence of the same gene mutations may have differing effects dependent upon the cellular background.

\section{Likely cell of origin of ChRCC}

As TCGA had previously carried out a comprehensive study of ccRCC [16] prior to our ChRCC study, we were able to compare the molecular profiles of these two types of RCC. From the perspective of multiple data platforms (copy number, whole exome, RNA sequencing, and DNA methylation), ChRCC appeared entirely distinct from ccRCC, which suggested that the two may arise from different cells of origin. In particular, we observed widespread differences in DNA methylation between ChRCC and ccRCC, involving over tens of thousands of genomic loci. In principle, some of these differences could involve cancer-relevant pathways, but other differences might also reflect the respective cells of origin of the two cancers [17]. Previously, ChRCC had been postulated to arise from intercalated cells in the distal 
convoluted tubule of the kidney nephron, while ccRCC could arise from cells in the proximal convoluted tubule. This theory is based on previous work focusing on tubulespecific protein markers, by immunohistochemistry [18], but we sought in our study to revisit this question, using a global analysis of gene expression.

In addressing the question of the cell of origin of ChRCC, we used a creative analytical approach, involving an external expression profiling dataset from Cheval et al. [19]. In the Cheval study, normal tissue, from both mouse and human nephron specimens, was carefully microdissected from the glomerulus and seven anatomically defined nephron segments and subjected to gene expression profiling. The Cheval study associated both global gene expression patterns and individual gene markers with specific sections of the nephron, and-to the benefit of future science--made the entire dataset publicly available, allowing us to analyze these data in the context of ChRCC. The actual analysis involving the TCGA and Cheval datasets was straightforward: For each gene in our TCGA kidney cancer dataset (combined ChRCC and ccRCC), expression values were centered across sample profiles (using the mean centroid of the two cancers); in a similar manner, within each of the human and mouse datasets from the Cheval study, values were centered across sample profiles. The respective centered datasets represented differential expression profiles, with essentially unitless values. For each TCGA and Cheval differential expression profile, we computed the global inter-profile correlation, using all $\sim 4000$ genes in common.

Our supervised analysis, globally comparing each TCGA ChRCC or ccRCC tumor expression profile to that of each sample in the Cheval nephron atlas, showed high mRNA expression correlations for ChRCC with distal regions of the nephron; ccRCC tumors demonstrated gene expression profiles, on the other hand, which correlated with patterns associated with the proximal nephron. These associations were strikingly clear, being observed in both the mouse and human nephron datasets. While our analysis associated ChRCC and ccRCC with a respective site of origin within the nephron, and not necessarily a specific cell of origin, when the previous immunohistochemistry studies of RCC are also taken into account [18], the best explanation to date for all of these data is that ChRCC and ccRCC do in fact have different cells of origin, as originally postulated. With this in mind, it is possible that therapies evolved for use on ccRCC tumors may not be successful in ChRCC.

Cancer cell of origin is a question relevant to many other cancers in addition to ChRCC. Knowledge of the cell of origin may provide insights into disease etiology, for example. Widespread molecular heterogeneity may be observed within other cancers sharing a common tissueof-origin $[20,21]$. In our study of RCC, we were able to put much of the observed molecular differences into some meaningful context, where the global gene expression differences had been previously noted [22] but without an overall framework to explain them. For our analytical approach to be applied to other cancers, a suitable molecular profiling dataset representing the candidate cells of origin would be needed, though in most cases, a suitable dataset may not be readily available. Recently, expression profiling data representing hundreds of cell types was made available by the FANTOM consortium [23], which data might be utilized in the analysis of other cancers, taking a similar approach to that of our use of the Cheval nephron dataset. In addition, as DNA methylation profiles of normal cell types become available in the future, such data might also be analyzed in the context of cancer.

\section{Metabolism and mitochondrial function in ChRCC}

For some time, kidney cancer has been viewed as essentially a metabolic disease [24-26]. Mutations in a number of kidney cancer-associated genes, including $V H L, M E T, F L C N, T S C 1, T S C 2, F H$, and $S D H$, result in dysregulation of metabolic pathways involved in oxygen, iron, energy or nutrient sensing. For example, germline mutations of $F H$, which lead to a metabolic shift to aerobic glycolysis, are associated with the development of the genetic syndrome of hereditary leiomyomatosis and renal cell cancer (HLRCC), which is characterized by an aggressive papillary type II renal cell carcinoma [27]. As another example, ccRCC is closely associated with $V H L$ gene alterations that lead to stabilization and resulting accumulation of HIF- $1 \alpha$ and HIF- $2 \alpha$; the increased HIF levels lead to both alterations in glutamine metabolism, and sensitivity to glutamine deprivation [28], as well as increased transcription of a number of downstream genes such as GLUT1, which enables transport of glucose for ATP production [24]. In TCGA's comprehensive molecular analysis of ccRCC, widespread molecular changes were associated with tumors having a poorer outcome; these changes implicated a metabolic shift, with tumors altering their usage of key pathways and metabolites in a "Warburg-like" effect. Worse survival in ccRCC correlated with up-regulation of pentose phosphate pathway genes and fatty acid synthesis genes, while better survival correlated with up-regulation of AMPK complex genes, multiple Krebs cycle genes, and PI3K pathway inhibitors (e.g. PTEN, TSC2) [16].

In direct contrast to ccRCC, previous studies using F-18-fluorodeoxyglucose PET/CT have suggested that ChRCC exhibits a non-glycolytic metabolic profile [29]. In addition, our ChRCC cases showed increased expression of genes involved in the Krebs cycle and the electron transport chain for generation of ATP[1]. Given the indicated prevalent role of mitochondria and metabolism in ChRCC, we sequenced mtDNA from 61 of our $66 \mathrm{ChRCC}$ cases, using an LR-PCR-based amplification approach. 
MtDNA gene mutations in ChRCC all involved complex I of the electron transport chain, in particular in the NADH dehydrogenase 5 gene (MT-ND5, in 6 of our 61 cases); due to the type of mutations observed, these would be likely to result in loss of complex I activity. Furthermore, $M T$ ND5-mutated ChRCC cases were significantly associated with eosinophilic histology, which itself was associated with a phenotype of fewer copy number alterations and distinctive gene expression patterns.

Further studies to dissect the precise role of mitochondria DNA alterations could shed light on how core metabolic pathways may be altered in ChRCC and other diseases. Renal oncocytoma, a benign renal tumor that may also arise from the distal nephron, shares several similarities with ChRCC, including abundant, eosinophilic cytoplasm and densely packed mitochondria[15, 30]. It is believed that oncocytomas and ChRCC represent two ends of a tumor spectrum that also includes BHD-associated hybrid-oncocytic tumors, all of which have been found to have high levels of expression of mitochondrial and oxidative phosphorylation genes and to cluster together based on this feature [31]. Mitochondrial accumulation in renal oncocytomas was initially hypothesized as a compensatory mechanism of inefficient oxidative phosphorylation [32]. More recently, isolated loss of complex I activity was identified and determined to result from homoplasmic, somatically acquired mutations in mitochondrial complex I genes [32-34]. Our findings and that of other studies would suggest an irregular metabolic program supporting the growth of $\mathrm{ChRCC}[1,29]$, one counter to the Warburg phenomenon observed commonly in ccRCC and many other cancers. However, elsewhere mutations in MT-ND5 and other complex I genes have been hypothesized to lead to inactivation of oxidative phosphorylation and reliance on glycolysis [35]. Some functional studies into the role MT-ND5 mutations have been carried out to date, including one by Hofhaus and Attardi [36], where loss of MT-ND5 and complex I activity was found to occur in cell lines that developed resistance to an inhibitor of oxidative phosphorylation, and another by Park et al.[37], where MT-ND5 mutations were associated with alteration of reactive oxygen species generation and apoptosis as well as with loss of oxidative phosphorylation.

For this review, we surveyed the expression of genes related to Krebs cycle and Electron Transport Chain (ETC), in an RNA-seq dataset of 3,564 cancers representing 12 different cancer types in addition to ChRCC and representing a range of tissues of origin. The resulting heat map is shown in Figure 1, where sample profiles were clustered on the basis of selected metabolism-related genes. We find that the samples tend to segregate on the basis of high or low average expression of these genes, though for some tumor types, two or more subgroups may be defined. In particular, the ChRCC ("KICH") cases form a tight and distinctive group, with the highest average expression of Krebs cycle and ETC genes relative to that of other tumor types. In contrast, ccRCC cases associate with multiple subgroups, on the basis of low to medium expression of Krebs cycle and ETC genes. From TCGA's previous ccRCC study, we know that the ccRCC cases with the lowest expression tend to be associated with worse patient outcome [16].

Additional pan-cancer studies to elucidate the role of metabolism and mitochondrial function in cancer could be undertaken, using genomic data resources from TCGA and elsewhere. While the LR-PCR method to sequence mtDNA in a large number of cancers could be fairly labor intensive, in our study we had the opportunity to compare mtDNA mutation results using either LR-PCRbased or WGS-based approaches [35], and we found these two to be highly complementary to each other. As the number of WGS profiles represented in TCGA is currently upwards of $\sim 1,000$ tumors [38], these data could be further mined for mtDNA mutations across various cancer types. One recent study by Larman et al. [35], surveyed mtDNA mutations in 226 tumors representing five types of cancer (colon adenocarcinoma, rectal adenocarcinoma, acute myeloid leukemia, glioblastoma, and ovarian serous cystadenocarcinoma) using WGS data, where $65 \%$ of somatic truncating mutations occurred in $M T-N D 5$, suggesting these alterations affecting electron transport chain may not be limited to one rare tumor type. As a future set of studies into metabolism in cancer, metabolomic profiling data could be generated, in addition to our using mRNA expression as a surrogate for metabolite levels. In addition, more functional studies into the role of mtDNA mutations are needed, examining their effects on several cancer types, where we might consider alternative roles for complex I alteration in addition to the initiation of a Warburg effect as observed in some cancers [39].

\section{TERT promoter alterations in ChRCC}

A highlight of our ChRCC study was the use of highcoverage WGS data covering the somatic and germline genomes (involving $\mathrm{n}=50 \mathrm{ChRCC}$ cases) to uncover structural rearrangements involving the TERT promoter region. This finding underscores the value of taking a comprehensive genomic approach to a particular cancer, where unanticipated discoveries may arise from any one avenue of investigation. We first noted that three our cases manifested strong patterns of kataegis, a phenomenon involving highly localized substitution mutations $(\mathrm{C}>\mathrm{T}$ or $\mathrm{C}>\mathrm{G})[12,40]$. The fact that this pattern was found in only a fraction of our cases invited us to compare ChRCC cases with and without kataegis. From RNA-seq analysis, the top most differentially expressed gene was TERT, which showed very high levels in kataegis cases as well as in a handful of additional cases. The question arose as to 

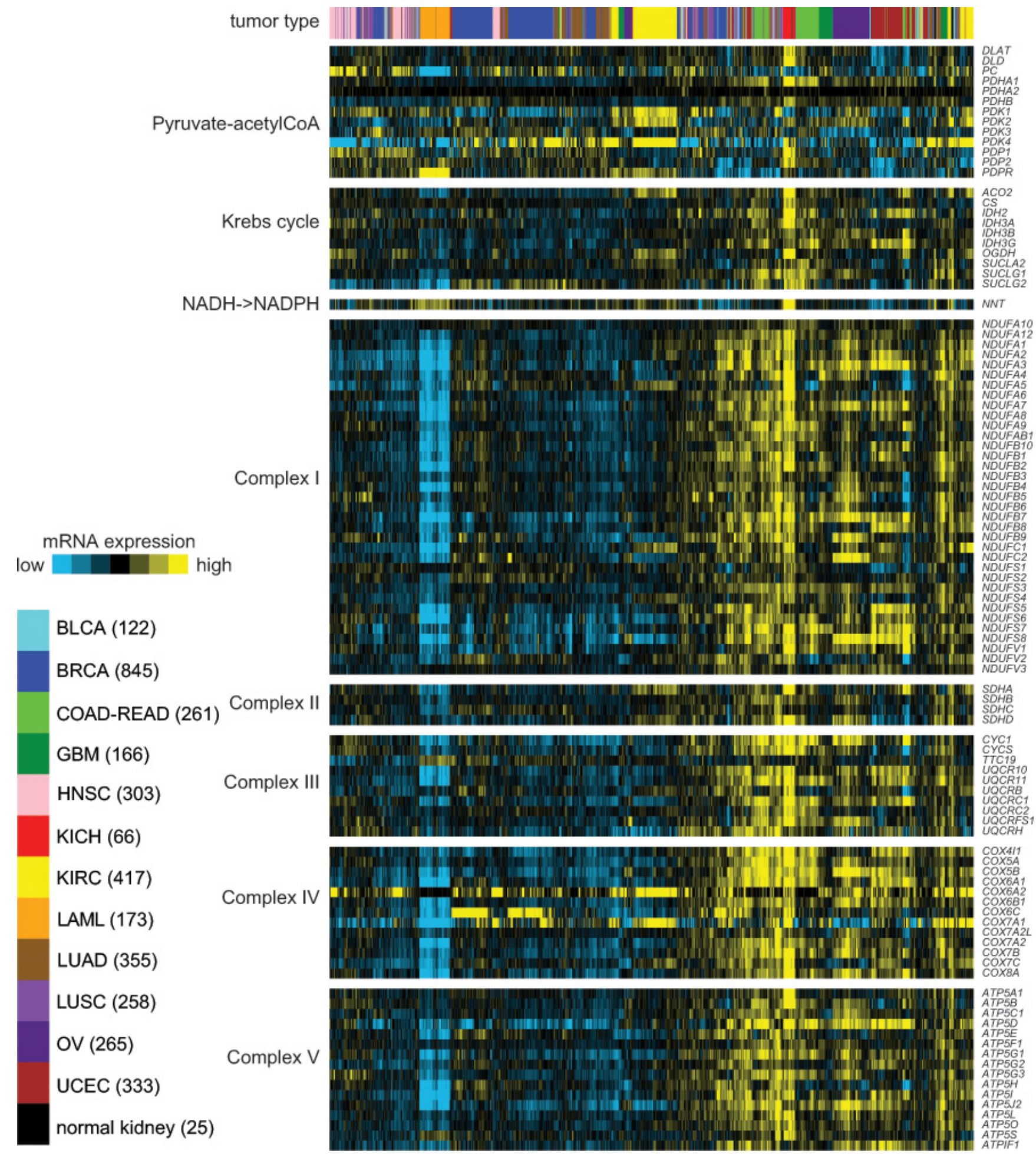

Figure 1: In a panel of human cancers of various tissues of origin (from TCGA, $n=3,564$ samples), differential expression (relative to sample median) of genes related to Krebs cycle and Electron Transport Chain (ETC). RNA-seq profiles are from the recent TCGA pan-cancer multiplatform subtyping analysis, with the addition of sample profiles of $\mathrm{ChRCC}(\mathrm{KICH})$ and normal kidney from TCGA ChRCC study [1]. On the basis of the expression patterns of the selected genes, samples were clustered by unsupervised hierarchical method [50]. KICH, chromophobe renal cell carcinoma (ChRCC); BLCA, bladder cancer; BRCA, breast cancer; COAD, colon cancer; GBM, glioblastoma; HNSC, head and neck squamous cell carcinoma; KIRC, clear cell renal cell carcinoma (ccRCC); LAML, acute myeloid leukemia; LUAD, lung adenocarcinoma; LUSC, lung squamous; OV, serous ovarian cancer; READ, rectal cancer; UCEC, endometrial cancer. 
how TERT could be highly expressed in only a subset of ChRCC, while being at very low or undetectable levels in most of the other cases. The cases with the highest TERT expression did not have the previously described activating promoter mutations (C228T and C250T) and did not have copy gains to the extent of being able to explain the corresponding increase in expression. However, an examination of copy number levels in the TERT promoter region identified abrupt changes in copy for six ChRCC cases, which was indicative of structural breakpoints. These breakpoints were first confirmed by WGS analysis using Meerkat algorithm [41], and then independently validated by PCR.

TERT encodes a rate-limiting catalytic subunit of telomerase that maintains genomic integrity. Over $90 \%$ of cancers show an up-regulation of the telomerase enzyme, which can occur by TERT over-expression as well as by other means [3]. Previously known mechanisms for TERT up-regulation include point mutations in the promoter [2, $3]$, viral genome integration [42], gene amplification [43, 44], and germline polymorphisms [45]. The promoter mutations $\mathrm{C} 228 \mathrm{~T}$ and $\mathrm{C} 250 \mathrm{~T}$, now observed in a wide variety of cancers (including bladder cancer, glioma, melanoma, squamous cell carcinoma, liver cancer, ovarian cancer, and thyroid cancer) $[3,38,46]$, create de novo Ets/ TCF binding sites, which can increase transcriptional activity from the promoter.

In this review, we surveyed TERT expression levels across $>3,500$ human cancers [20], including ChRCC (Figure 2). As with ChRCC, TERT mRNA levels vary widely within other cancer types, from undetectable to thousands of units by RNA-seq. Interestingly, for ChRCC, the somatically altered TERT subset defined the group of tumors displaying elevated TERT expression, with the

- C228T mutation $-\mathrm{C} 250 \mathrm{~T}$ mutation $\bullet$ promoter SV

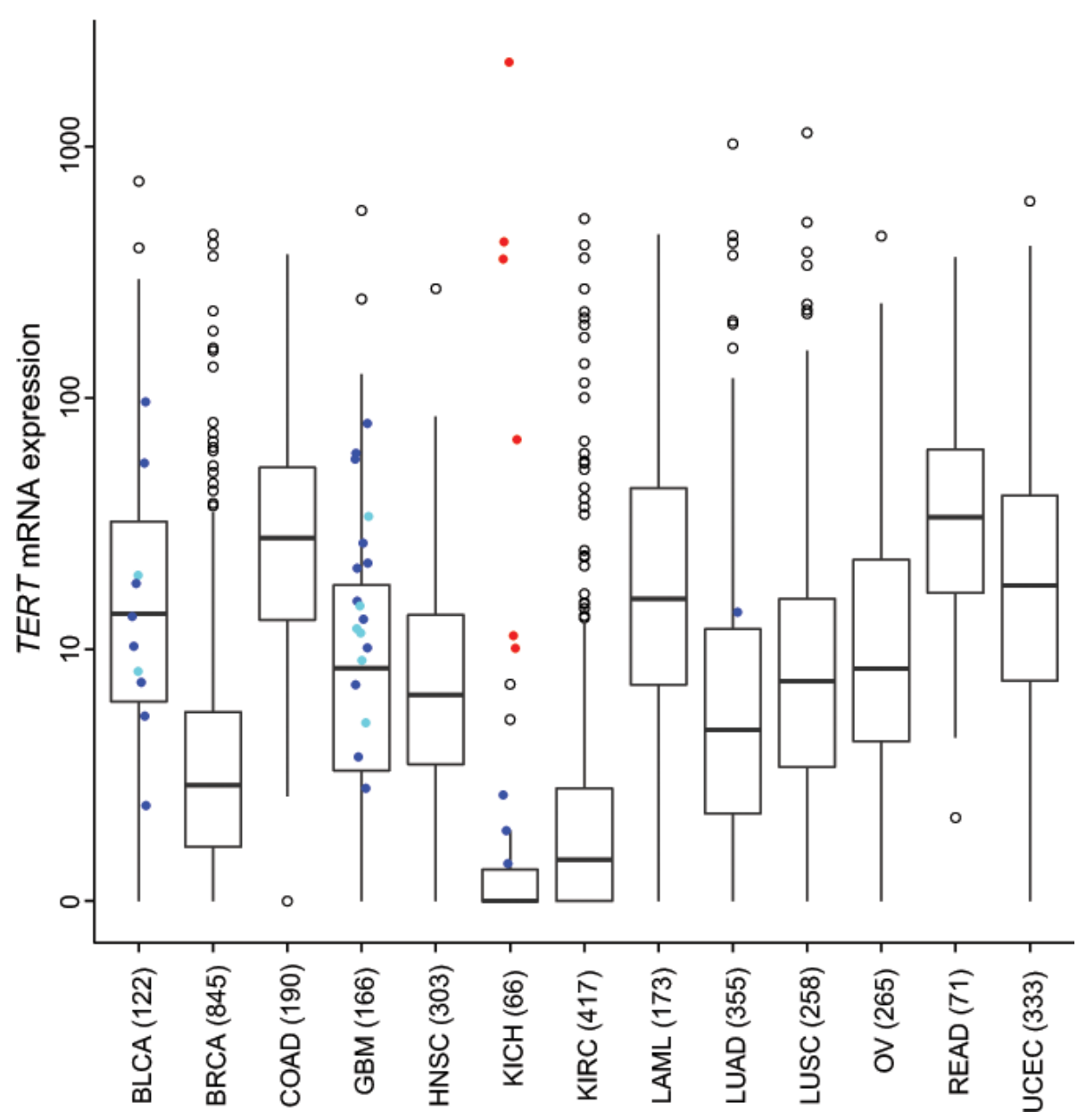

Figure 2: In a panel of human cancers of various tissues of origin (from TCGA, $\mathbf{n}=3$,564 samples), TERT mRNA expression levels by cancer type. Also indicated are those cases previously found to harbor a TERT promoter mutation (C228T or $\mathrm{C} 250 \mathrm{~T})$ by WGS analysis ( $\mathrm{n}=555$ human tumors) $[1,46]$, as well as the ChRCC (KICH) cases for which a structural variant (SV) breakpoint within the TERT promoter region was found [1]. For Box plots, boxes represent 25\% (Q1), median, and 75\% (Q3), and the upper whisker is the most extreme value that is no more than Q3+1.5 IQR and the lower whisker is the most extreme value that is no less than Q1-1.5 IQR. See Figure 1 legend for tumor type designations. 
majority of remaining tumors displaying quite low levels. For a subset of the cancers in Figure 2, WGS data were available, allowing us to note which cases were previously found to harbor the C228T or C250T mutations [46]. For the cancers surveyed here, TERT promoter mutations were mainly found within bladder cancers and glioblastomas, as well as for three of our ChRCC cases. While the point mutations tend to associate with higher TERT expression, most of the six ChRCC cases with promoter-associated structural variants (SVs) appear even higher than most cancers with the mutation. For most of the tumor cases outside of ChRCC represented in Figure 2, WGS data are not available, thus the status of TERT promoter alteration is currently unknown. To date, only in ChRCC cases have promoter SVs been thus far identified, and a survey of other cancer types for structural variants impacting TERT remains to be carried out.

To the best of our knowledge, the only instance of this phenomenon of TERT promoter SVs being observed previous to our study, was in a report by Zhao et al. [47] of non-tumorigenic, cultured fibroblast cells undergoing immortalization, which results provides some relevant context for our own findings. Zhao et al. studied TERT activation using genetically related pairs of telomerasenegative $(\operatorname{Tel}(-))$ and -positive $(\operatorname{Tel}(+))$ fibroblast lines. TERT was found translocated in all three Tel $(+)$ cell lines examined but not in their parental pre-crisis cells and Tel(-) immortal siblings, with the breakage points being mapped to regions upstream of the TERT promoter. In light of the Zhao study, the observed TERT promoter rearrangements in our ChRCC cases may result from genomic instability in precancerous cells undergoing the crisis stage of immortalization, leading to activated telomerase. This hypothesis would be consistent with our ChRCC WGS analysis examining intra-tumor heterogeneity, where in most cases the TERT promoter SVs were estimated to reside in nearly all of the tumor cells, indicating that these rearrangements represent early events in the cancer.

While the TERT promoter-associated SVs were found with high recurrence in ChRCC and strongly associated with up-regulation of the gene, the mechanism of how this up-regulation occurs remains to be elucidated. In our ChRCC study, we carried out a systematic search for any cis-regulatory elements that might be commonly involved with the observed TERT promoter-associated SVs. In many cases, we did observe a number of cisregulatory elements being placed in close proximity to the core promoter of TERT, as a result of rearrangement, although these results as yet do not lead us to a precise mechanism. Also, in the Zhao study [47], the authors had put forth the idea that TERT is normally silenced in noncancerous cells, but that rearrangements upstream of TERT may allow the promoter to escape the repressive chromatin environment and thereby activate telomerase expression; we examined our TCGA DNA methylation data for any evidence of this in ChRCC. From our data, however, it was not clear that TERT is usually silenced in normal tissues, and we did not observe noticeable differences between the TERT-rearranged ChRCC samples and the rest. The caveat here is that our HM450 platform may not have the resolution to fully study the DNA methylation pattern around break points, as many of the involved regions are intergenic and have no coverage.

\section{Perspectives and Future Work: Pan-cancer analyses beyond the exome}

To date, most large scale sequencing studies in cancer have largely focused on mutations within the exome $[48,49]$, which comprises on the order of $1 \%$ of the genome. Our ChRCC study demonstrates the utility of searching for potential driver alterations outside of the exome, e.g. through WGS and mtDNA profiling. For genomic datasets generated as part of efforts by TCGA and by the International Cancer Genomics Consortium (ICGC) [48], a large number of samples now have WGS data available, which data could and should be more deeply mined. A recent study by Weinhold et al. [38] analyzed WGS data from 863 human cancers of various types (most of these being from TCGA), in order to systematically identify noncoding regions that were recurrently mutated, revealing several novel regulatory changes. Future pancancer studies of whole genomes could and should include analysis of structural variants, which can have major effects on gene expression. Further, the analysis of mtDNA mutations lends further support for studies exploring this often overlooked segment of the genome. Our comprehensive molecular datasets on ChRCC should continue to serve as a resource for comparisons with future pan-cancer studies, or as a source for new areas to explore in cancer biology as well as for setting the stage for further investigations that lead to precision therapy directed toward the treatment of ChRCC.

\section{GRANT SUPPORT}

This study was supported in part by P30 CA125123 (CJC) and 5U24CA143843 (CJC) from the National Institute of Health and by RP120713 (CJC) from the Cancer Prevention \& Research Institute of Texas (CPRIT)

\section{CONFLICT OF INTEREST}

The author declares no conflicts of interest.

\section{Abbreviations}

ChRCC, chromophobe renal cell carcinoma; ccRCC, clear cell renal cell carcinoma; TCGA, The Cancer Genome Atlas; RNA-seq, RNA sequencing; mtDNA, 
mitochondrial DNA; WGS, whole genome sequencing

\section{REFERENCES}

1. Davis C, Ricketts C, Wang M, Yang L, Cherniack A, Shen H, Buhay C, Kang H, Kim S, Fahey C, Hacker K, Bhanot G, Gordenin D, Chu A, Gunaratne P, Biehl M, et al. The somatic genomic landscape of chromophobe renal cell carcinoma. Cancer Cell. 2014; 26(3):319-330.

2. Huang FW, Hodis E, Xu MJ, Kryukov GV, Chin L and Garraway LA. Highly recurrent TERT promoter mutations in human melanoma. Science. 2013; 339(6122):957-959.

3. Heidenreich B, Rachakonda P, Hemminki K and Kumar R. TERT promoter mutations in cancer development. Curr Opin Genet Dev. 2013; 24C:30-37.

4. Storkel S, Eble JN, Adlakha K, Amin M, Blute ML, Bostwick DG, Darson M, Delahunt B and Iczkowski K. Classification of renal cell carcinoma: Workgroup No. 1. Union Internationale Contre le Cancer (UICC) and the American Joint Committee on Cancer (AJCC). Cancer. 1997; 80(5):987-989.

5. Amin MB, Amin MB, Tamboli P, Javidan J, Stricker H, de-Peralta Venturina M, Deshpande A and Menon M. Prognostic impact of histologic subtyping of adult renal epithelial neoplasms: an experience of 405 cases. Am J Surg Pathol. 2002; 26(3):281-291.

6. Przybycin CG, Cronin AM, Darvishian F, Gopalan A, AlAhmadie HA, Fine SW, Chen YB, Bernstein M, Russo P, Reuter VE and Tickoo SK. Chromophobe renal cell carcinoma: a clinicopathologic study of 203 tumors in 200 patients with primary resection at a single institution. Am J Surg Pathol. 2011; 35(7):962-970.

7. Speicher MR, Schoell B, du Manoir S, Schrock E, Ried T, Cremer T, Storkel S, Kovacs A and Kovacs G. Specific loss of chromosomes 1, 2, 6, 10, 13, 17, and 21 in chromophobe renal cell carcinomas revealed by comparative genomic hybridization. Am J Pathol. 1994; 145(2):356-364.

8. Schmidt LS, Warren MB, Nickerson ML, Weirich G, Matrosova V, Toro JR, Turner ML, Duray P, Merino M, Hewitt S, Pavlovich CP, Glenn G, Greenberg CR, Linehan WM and Zbar B. Birt-Hogg-Dube syndrome, a genodermatosis associated with spontaneous pneumothorax and kidney neoplasia, maps to chromosome 17p11.2. Am J Hum Genet. 2001; 69(4):876-882.

9. Nagy A, Zoubakov D, Stupar Z and Kovacs G. Lack of mutation of the folliculin gene in sporadic chromophobe renal cell carcinoma and renal oncocytoma. Int J Cancer. 2004; 109(3):472-475.

10. Gad S, Lefevre SH, Khoo SK, Giraud S, Vieillefond A, Vasiliu V, Ferlicot S, Molinie V, Denoux Y, Thiounn N, Chretien Y, Mejean A, Zerbib M, Benoit G, Herve JM, Allegre G, et al. Mutations in BHD and TP53 genes, but not in HNF1beta gene, in a large series of sporadic chromophobe renal cell carcinoma. Br J Cancer. 2007;
96(2):336-340.

11. Shuch B, Ricketts CJ, Vocke CD, Komiya T, Middelton LA, Kauffman EC, Merino MJ, Metwalli AR, Dennis P and Linehan WM. Germline PTEN Mutation Cowden Syndrome: An Under-Appreciated Form of Hereditary Kidney Cancer. The Journal of urology. 2013.

12. Alexandrov LB, Nik-Zainal S, Wedge DC, Aparicio SA, Behjati S, Biankin AV, Bignell GR, Bolli N, Borg A, Borresen-Dale AL, Boyault S, Burkhardt B, Butler AP, Caldas C, Davies HR, Desmedt C, et al. Signatures of mutational processes in human cancer. Nature. 2013; 500(7463):415-421.

13. Cancer Genome Atlas N. Comprehensive molecular portraits of human breast tumours. Nature. 2012; 490(7418):61-70.

14. Thoenes W, Storkel S and Rumpelt HJ. Human chromophobe cell renal carcinoma. Virchows Archiv B, Cell pathology including molecular pathology. 1985; 48(3):207-217.

15. Tickoo SK, Lee MW, Eble JN, Amin M, Christopherson T, Zarbo RJ and Amin MB. Ultrastructural observations on mitochondria and microvesicles in renal oncocytoma, chromophobe renal cell carcinoma, and eosinophilic variant of conventional (clear cell) renal cell carcinoma. Am J Surg Pathol. 2000; 24(9):1247-1256.

16. The_Cancer_Genome_Atlas_Research_Network. Comprehensive molecular characterization of clear cell renal cell carcinoma. Nature. 2013; 499(7456):43-49.

17. Shen $\mathrm{H}$ and Laird PW. Interplay between the cancer genome and epigenome. Cell. 2013; 153(1):38-55.

18. Prasad SR, Narra VR, Shah R, Humphrey PA, Jagirdar J, Catena JR, Dalrymple NC and Siegel CL. Segmental disorders of the nephron: histopathological and imaging perspective. The British journal of radiology. 2007; 80(956):593-602.

19. Cheval L, Pierrat F, Rajerison R, Piquemal D and Doucet A. Of mice and men: divergence of gene expression patterns in kidney. PloS one. 2012; 7(10):e46876.

20. Hoadley K, Yau C, Wolf D, Cherniack A, Tamborero D, $\mathrm{Ng}$ S, Leiserson M, Niu B, McLellan M, Uzunangelov V, Zhang J, Kandoth C, Akbani R, Shen H, Omberg L, Chu A, et al. Multiplatform Analysis of 12 Cancer Types Reveals Molecular Classification within and across Tissues of Origin. Cell. 2014; 158(4):929-944.

21. Perou C, Sørlie T, Eisen M, van de Rijn M, Jeffrey S, Rees C, Pollack J, Ross D, Johnsen H, Akslen L, Fluge O, Pergamenschikov A, Williams C, Zhu S, Lønning P, Børresen-Dale A, et al. Molecular portraits of human breast tumours. Nature. 2000; 406(6797):747-752.

22. Williams A, Higgins J, Zhao H, Ljunberg B and Brooks J. CD 9 and vimentin distinguish clear cell from chromophobe renal cell carcinoma. BMC Clin Pathol. 2009; 9:9.

23. FANTOM_Consortium_and_the_RIKEN_PMI_and CLST_(DGT), Forrest A, Kawaji H, Rehli M, Baillie J, de 
Hoon M, Lassmann T, Itoh M, Summers K, Suzuki H, Daub C, Kawai J, Heutink P, Hide W, Freeman T, Lenhard B, et al. A promoter-level mammalian expression atlas. Nature. 2014; 507(7493):462-470.

24. Linehan W, Srinivasan R and Schmidt L. The genetic basis of kidney cancer: a metabolic disease. Nat Rev Urol. 2010; 7(5):277-285.

25. Linehan W and Rathmell W. Kidney cancer. Urol Oncol. 2012; 30(6):948-951.

26. Linehan $\mathrm{W}$ and Ricketts $\mathrm{C}$. The metabolic basis of kidney cancer. Semin Cancer Biol. 2013; 23(1):46-55.

27. Tong W, Sourbier C, Kovtunovych G, Jeong S, Vira M, Ghosh M, Romero V, Sougrat R, Vaulont S, Viollet B, Kim Y, Lee S, Trepel J, Srinivasan R, Bratslavsky G, Yang Y, et al. The glycolytic shift in fumarate-hydratase-deficient kidney cancer lowers AMPK levels, increases anabolic propensities and lowers cellular iron levels. Cancer Cell. 2011; 20(3):315-327.

28. Gameiro P, Yang JM, AM, Pérez-Carro R, Baker R, Wang Z, Arreola A, Rathmell W, Olumi A, López-Larrubia P, Stephanopoulos G and Iliopoulos O. In vivo HIF-mediated reductive carboxylation is regulated by citrate levels and sensitizes VHL-deficient cells to glutamine deprivation. Cell Metab. 2013; 17(3):372-385.

29. Ho C, Chen S, Ho K, Chan W, Leung Y, Cheng K, Wong $\mathrm{K}$, Cheung $\mathrm{M}$ and Wong K. Dual-tracer PET/CT in renal angiomyolipoma and subtypes of renal cell carcinoma. Clin Nucl Med. 2012; 37(11):1075-1082.

30. Amin MB, Paner GP, Alvarado-Cabrero I, Young AN, Stricker HJ, Lyles RH and Moch H. Chromophobe renal cell carcinoma: histomorphologic characteristics and evaluation of conventional pathologic prognostic parameters in 145 cases. Am J Surg Pathol. 2008; 32(12):1822-1834.

31. Klomp JA, Petillo D, Niemi NM, Dykema KJ, Chen J, Yang XJ, Saaf A, Zickert P, Aly M, Bergerheim U, Nordenskjold M, Gad S, Giraud S, Denoux Y, Yonneau L, Mejean A, et al. Birt-Hogg-Dube renal tumors are genetically distinct from other renal neoplasias and are associated with up-regulation of mitochondrial gene expression. BMC medical genomics. 2010; 3:59.

32. Simonnet $\mathrm{H}$, Demont J, Pfeiffer $\mathrm{K}$, Guenaneche L, Bouvier R, Brandt $\mathrm{U}$, Schagger $\mathrm{H}$ and Godinot $\mathrm{C}$. Mitochondrial complex I is deficient in renal oncocytomas. Carcinogenesis. 2003; 24(9):1461-1466.

33. Gasparre G, Hervouet E, de Laplanche E, Demont J, Pennisi LF, Colombel M, Mege-Lechevallier F, Scoazec JY, Bonora E, Smeets R, Smeitink J, Lazar V, Lespinasse J, Giraud S, Godinot C, Romeo G, et al. Clonal expansion of mutated mitochondrial DNA is associated with tumor formation and complex I deficiency in the benign renal oncocytoma. Hum Mol Genet. 2008; 17(7):986-995.

34. Mayr JA, Meierhofer D, Zimmermann F, Feichtinger R, Kogler C, Ratschek M, Schmeller N, Sperl W and Kofler B. Loss of complex I due to mitochondrial DNA mutations in renal oncocytoma. Clin Cancer Res. 2008; 14(8):22702275 .

35. Larman TC, DePalma SR, Hadjipanayis AG, Cancer Genome_Atlas_Research_Network, Protopopov A, Zhang J, Gabriel SB, Chin L, Seidman CE, Kucherlapati $\mathrm{R}$ and Seidman JG. Spectrum of somatic mitochondrial mutations in five cancers. Proc Natl Acad Sci U S A. 2012; 109(35):14087-14091.

36. Hofhaus G and Attardi G. Efficient selection and characterization of mutants of a human cell line which are defective in mitochondrial DNA-encoded subunits of respiratory NADH dehydrogenase. Mol Cell Biol. 1995; 15(2):964-974.

37. Park J, Sharma L, Li H, Xiang R, Holstein D, Wu J, Lechleiter J, Naylor S, Deng J, Lu J and Bai Y. A heteroplasmic, not homoplasmic, mitochondrial DNA mutation promotes tumorigenesis via alteration in reactive oxygen species generation and apoptosis. Hum Mol Genet. 2009; 18(9):1578-1589.

38. Weinhold N, Jacobsen A, Schultz N, Sander C and Lee W. Genome-wide analysis of noncoding regulatory mutations in cancer. Nature genetics. 2014; 46(11):1160-1165.

39. Ward P and Thompson C. Metabolic reprogramming: a cancer hallmark even warburg did not anticipate. Cancer Cell. 2012; 21(3):297-308.

40. Nik-Zainal S, Alexandrov LB, Wedge DC, Van Loo P, Greenman CD, Raine K, Jones D, Hinton J, Marshall J, Stebbings LA, Menzies A, Martin S, Leung K, Chen L, Leroy C, Ramakrishna M, et al. Mutational processes molding the genomes of 21 breast cancers. Cell. 2012; 149(5):979-993.

41. Yang L, Luquette L, Gehlenborg N, Xi R, Haseley P, Hsieh C, Zhang C, Ren X, Protopopov A, Chin L, Kucherlapati $\mathrm{R}$, Lee $\mathrm{C}$ and Park P. Diverse mechanisms of somatic structural variations in human cancer genomes. Cell. 2013; 153(4):919-929.

42. Totoki Y, Tatsuno K, Covington K, Ueda H, Creighton C, Kato M, Tsuji S, Donehower L, Slagle B, Nakamura H, Yamamoto S, Shinbrot E, Hama N, Lehmkuhl M, Hosoda F, Arai Y, et al. Trans-ancestry mutational landscape of hepatocellular carcinoma genomes. Nature genetics. 2014; 46(12):1267-1273.

43. Y C, Adams A, Robbins P, Lin Q and Khavari P. Use of human tissue to assess the oncogenic activity of melanomaassociated mutations. Nat Genet. 2005; 37(7):745-749.

44. Weir B, Woo M, Getz G, Perner S, Ding L, Beroukhim R, Lin W, Province M, Kraja A, Johnson L, Shah K, Sato M, Thomas R, Barletta J, Borecki I, Broderick S, et al. Characterizing the cancer genome in lung adenocarcinoma. Nature. 2007; 450(7171):893-898.

45. Rafnar T, Sulem P, Stacey S, Geller F, Gudmundsson J, Sigurdsson A, Jakobsdottir M, Helgadottir H, Thorlacius S, Aben K, Blöndal T, Thorgeirsson T, Thorleifsson G, Kristjansson K, Thorisdottir K, Ragnarsson R, et al. 
Sequence variants at the TERT-CLPTM1L locus associate with many cancer types. Nature genetics. 2009; 41(2):221227.

46. Fredriksson N, Ny L, Nilsson J and Larsson E. Systematic analysis of noncoding somatic mutations and gene expression alterations across 14 tumor types. Nature genetics. 2014; 46(12):1258-1263.

47. Zhao Y, Wang S, Popova E, Grigoryev S and Zhu J. Rearrangement of upstream sequences of the hTERT gene during cellular immortalization. Genes Chromosomes Cancer. 2009; 48(11):963-974.
48. International_Cancer_Genome_Consortium. International network of cancer genome projects. Nature. 2010; 464(7291):993-998.

49. Cancer_Genome_Atlas_Research_Network, Weinstein J, Collisson E, Mills G, Shaw K, Ozenberger B, Ellrott K, Shmulevich I, Sander C and Stuart J. The Cancer Genome Atlas Pan-Cancer analysis project. Nature genetics. 2013; 45(10):1113-1120.

50. Eisen MB, Spellman PT, Brown PO and Botstein D. Cluster analysis and display of genome-wide expression patterns. Proc Natl Acad Sci USA. 1998; 95:14863-14868. 